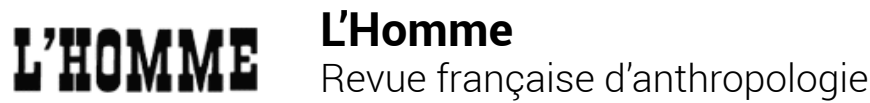

185-186 | 2008

L'anthropologue et le contemporain : autour de Marc Augé

\section{Actes de la recherche en sciences sociales, 2006, 164. Économies de la recherche}

Paris, Le Seuil, 125 p.

\section{Corinne Delmas}

\section{OpenEdition \\ Journals}

Édition électronique

URL : http://journals.openedition.org/lhomme/18512

DOI : 10.4000/lhomme.18512

ISSN : $1953-8103$

Éditeur

Éditions de l'EHESS

Édition imprimée

Date de publication : 1 janvier 2008

Pagination : $535-537$

ISSN : 0439-4216

Référence électronique

Corinne Delmas, «Actes de la recherche en sciences sociales, 2006, 164. Économies de la recherche», L'Homme [En ligne], 185-186 | 2008, mis en ligne le 20 mai 2008, consulté le 24 septembre 2020. URL : http://journals.openedition.org/lhomme/18512 ; DOI : https://doi.org/10.4000//homme.18512

Ce document a été généré automatiquement le 24 septembre 2020.

(c) École des hautes études en sciences sociales 


\title{
Actes de la recherche en sciences sociales, 2006, 164. Économies de la recherche
}

Paris, Le Seuil, 125 p.

\author{
Corinne Delmas
}

1 À L'HEURE OÙ l'enseignement supérieur et la recherche font l'objet, dans les pays européens, de réformes importantes, ce numéro des Actes de la recherche en sciences sociales est opportunément consacré aux "économies de la recherche». La courte présentation du dossier par Julien Duval et Johan Heilbron donne le ton, rappelant le " caractère autodestructeur d'une commercialisation sans bornes » (p. 7) et insistant sur le postulat selon lequel «l'autonomie (relative) de la recherche n'est pas simplement un enjeu corporatiste; c'est un fondement essentiel de la science, une condition à l'avancée des savoirs » (p. 9). Les auteurs soulignent combien « les effets qui s'observent aux États-Unis [en particulier dans le domaine de la recherche biomédicale et pharmaceutique] traduisent des contradictions et des dangers inhérents à une politique qui, en introduisant massivement dans la recherche publique des logiques issues du champ économique, risquent de détruire les structures dont elle veut se servir» (p. 9). Les cinq articles constituant ce dossier analysent les mécanismes et les croyances qui seraient spécifiques aux champs universitaire et scientifique. Paul Wouters étudie ainsi la "naissance du Science Citation Index », "aux origines de la scientométrie » et montre que le principe consistant à indexer les citations n'est pas une création «naturelle» de l'univers scientifique. Ce principe, initialement appliqué aux États-Unis à la littérature juridique a été transposé à la science par Eugene Garfield, entrepreneur spécialisé dans le domaine de l'information. La création du sci et le développement de la scientométrie ont été marqués par le contexte technologique et politique de la fin des années 1950 qui ont contribué à légitimer cette création. Erwan Lamy et Terry Shinn s'intéressent pour leur part à « l'autonomie scientifique face à la mercantilisation » à partir d'une étude des «formes d'engagement entrepreneurial des chercheurs en France ». L'enquête, menée auprès de 41 chercheurs créateurs 
d'entreprises, permet à ces auteurs de distinguer trois formes d'implication entrepreneuriale, celles des "académiques", des "pionniers" et des "janus", qui constituent trois types de réponses à cette mercantilisation sans être pour autant synonymes, selon les auteurs, d'un abandon des normes académiques. L'article de Sylvia Faure et Charles Soulié sur «la recherche universitaire à l'épreuve de la massification" a pour objectif de "comparer les pratiques de recherche selon les disciplines, en tenant compte des évolutions récentes de l'université»(p.61). Cet article rappelle les différenciations entre les lettres, les sciences humaines et sociales et les sciences en termes de recrutement, financement de la recherche ou " habitus de recherche » (p. 67) et conclut sur un paradoxe : «ce sont les disciplines traditionnelles, mais aussi les plus théoriques, dont le recrutement social et scolaire comme le prestige académique sont encore relativement élevés, qui sont en perte de vitesse démographique et n'ont plus la faveur des étudiants ni des financeurs, et qui par conséquent voient leur conception de l'université, de la recherche et de la connaissance relativisée, la crise des vocations scientifiques alimentant fortement ce processus de délégitimation » (p. 72). C'est « la définition légitime des sciences sociales au prisme du débat sur la crise de l'édition SHs » que l'article de Bruno Auerbach analyse pour sa part. Selon l'auteur, le véritable enjeu de ce thème de la "crise » se situe autour du hiatus entre l'offre professionnelle des chercheurs et les attentes des éditeurs. L'article de John B. Thompson sur «l'édition savante à la croisée des chemins » en analyse les évolutions en Grande-Bretagne et aux États-Unis au cours des trente dernières années ; il montre que les changements technologiques liés à la révolution numérique ne sont qu'un aspect de ces évolutions aux côtés de l'accélération du rythme des nouvelles éditions et de la course en avant d'éditeurs dépensant « beaucoup de temps et d'énergie pour maintenir leurs manuels à succès dans ce qui est virtuellement un état de révision permanente. Il ne s'agit pas d'un choix mais d'une nécessité : face à un marché de l'occasion florissant, la révision permanente est la seule manière d'éviter que votre capital ne perde de sa valeur très rapidement» (p.96). "Cinq propositions pour l'édition savante » concluent curieusement cet article.

2 L'article, particulièrement intéressant, d'Yves Gingras et Brigitte Gemme sur «l'emprise du champ scientifique sur le champ universitaire et ses effets » (pp. 50-60) montre combien les différentes prises de position dans les débats sur la réforme des études supérieures ne peuvent se comprendre que si on observe qu'ils sont implicitement fondés sur une confusion de la logique du champ universitaire avec celle du champ scientifique. Les tentatives récentes de transformation des programmes universitaires et l'orientation professionnelle des étudiants formés à la recherche correspondent en fait à une autonomisation accrue du champ universitaire par rapport au champ scientifique qui voit décliner son quasi-monopole en matière de définition des programmes de formation des $2^{\mathrm{e}}$ et $3^{\mathrm{e}}$ cycles. Les effets de cette interaction forte, aujourd'hui remise en cause, sont précisément analysés : imposition par le champ scientifique de ses problématiques dominantes en formation doctorale, vision déformée de la réalité du marché de l'emploi qui en résulte pour les étudiants et les professeurs, avec une tendance à survaloriser les carrières universitaires par rapport aux postes réellement accessibles - cette préférence des carrières universitaires pour les jeunes docteurs résultant également "d'un processus de socialisation centrée, pour des raisons historiques, sur le monde universitaire» (p.52)-, sentiments d'échec importants chez les diplômés qui n'occuperont pas le poste académique convoité importance relative accordée aux différents critères d'embauche des enseignants- 
chercheurs qui sont essentiellement de trois ordres - qualités de la recherche, capacités pédagogiques et personnalité d'un futur collègue pouvant partager les différentes tâches d'un département - avec une tendance à la valorisation du critère scientifique au détriment du critère pédagogique. L'article invite en conclusion à déplacer la focale des mutations des pratiques de recherche et de publication des enseignants-chercheurs vers celles affectant la formation des nouveaux chercheurs, en particulier la diversification des projets professionnels chez les doctorants dont les travaux se font en collaboration avec des organismes non universitaires: "Il faut toutefois être prudent et éviter de tracer des liens de causalité trop simples : il ne va pas de soi que c'est la participation à un projet de recherche coopérative qui amène l'étudiante ou l'étudiant à changer sa préférence professionnelle d'un poste universitaire à un poste non universitaire. Il est même très probable que le lien soit en partie l'inverse : en créant un espace légitime pour la formation à la recherche fondée sur des problèmes issus de l'extérieur du champ scientifique, les nouvelles formes d'encadrement des doctorants, en particulier les bourses pour la recherche en "milieu de pratique", incitent peut-être de nouvelles catégories d'étudiants à suivre leur formation de recherche, qu'il s'agisse de jeunes en formation initiale ou de professionnels déjà actifs sur le marché du travail et désireux d'acquérir de nouvelles qualifications » (p. 59).

3 Il faut souligner l'intérêt, à l'heure de la « réforme » des universités et de la recherche, de ce numéro des Actes de la recherche en sciences sociales. On aurait certes aimé que le statut de la science, les liens entre recherche savante et utilité sociale soient davantage explicités ; la citation de Charles Pierce, mise en quatrième de couverture - « La science véritable est l'étude des choses inutiles »- n'est pas directement traitée dans ce numéro qui n'en éclaire pas moins certaines évolutions contemporaines.

\section{AUTEUR}

\section{CORINNE DELMAS}

CNRS, Université Lille-II, Centre d'études et de recherches administratives, politiques et sociales, Lille.

corinne.delmas@univ-lille2.fr 